\title{
A simple proof of Pitman-Yor's Chinese restaurant process from its stick-breaking representation
}

https://doi.org/10.1515/demo-2019-0003

Received October 15, 2018; accepted February 1, 2019

\begin{abstract}
For a long time, the Dirichlet process has been the gold standard discrete random measure in Bayesian nonparametrics. The Pitman-Yor process provides a simple and mathematically tractable generalization, allowing for a very flexible control of the clustering behaviour. Two commonly used representations of the Pitman-Yor process are the stick-breaking process and the Chinese restaurant process. The former is a constructive representation of the process which turns out very handy for practical implementation, while the latter describes the partition distribution induced. Obtaining one from the other is usually done indirectly with use of measure theory. In contrast, we propose here an elementary proof of Pitman-Yor's Chinese Restaurant process from its stick-breaking representation.
\end{abstract}

Keywords: Bayesian nonparametrics, clustering, Pitman-Yor process, partitions, stick-breaking process

MSC: 62F15, 97K50

\section{Introduction}

The Pitman-Yor process defines a rich and flexible class of random probability measures which was developed by Perman et al. [26] and further investigated by Pitman [28], Pitman and Yor [30]. It is a simple generalization of the Dirichlet process [15], whose mathematical tractability contributed to its popularity in machine learning theory [7], probabilistic models for linguistic applications [35, 37], excursion theory [26, 30], measure-valued diffusions in population genetics [14, 27], combinatorics [19, 36] and statistical physics [11].

Its most prominent role is perhaps in Bayesian nonparametric statistics where it is used as a prior distribution, following the work of [17]. Applications in this setting embrace a variety of inferential problems, including species sampling [3, 12, 24], survival analysis and graphical models in genetics [18, 25], image segmentation [34], curve estimation [6], exchangeable feature allocations [5] and time-series and econometrics $[4,7]$.

Last but not least, the Pitman-Yor process is also employed in the context of nonparametric mixture modeling, thus generalizing the celebrated Dirichlet process mixture model of [21]. Nonparametric mixture models based on the Pitman-Yor process are characterized by a more flexible parameterization than the Dirichlet process mixture model, thus allowing for a better control of the clustering behaviour [9]. In addition, see $[2,13,17]$ for posterior sampling algorithms, $[23,32]$ for asymptotic properties, and $[6,31]$ for spike-and-slab extensions. If a standard assumption in multivariate model-based clustering is to model the intra-group distribution with independent Gaussian distribution (diagonal covariance), non independent components have also been proposed in order to better account for complex dependence structures [10, 20].

^Corresponding Author: Julyan Arbel: Univ. Grenoble Alpes, Inria, CNRS, LJK, 38000 Grenoble, France, E-mail: julyan.arbel@inria.fr

Caroline Lawless: Univ. Grenoble Alpes, Inria, CNRS, LJK, 38000 Grenoble, France 
It is well known that the Pitman-Yor process admits a stick-breaking representation [26, 30] that generalizes that of the Dirichlet process with iid stick breaks [33] to independent stick breaks. More specifically, let $\boldsymbol{v}_{i} \stackrel{\text { ind }}{\sim} \operatorname{Beta}(1-d, \alpha+i d)$ for $i=1,2, \ldots$ with $d \in(0,1)$ called the discount parameter and $\alpha>-d$ called the concentration parameter. Let $\left(\pi_{1}, \pi_{2}, \ldots\right)$ be the successive parts obtained in breaking a stick of length one according to $\boldsymbol{\pi}_{1}=\boldsymbol{v}_{1}$, and $\boldsymbol{\pi}_{j}=\boldsymbol{v}_{j} \prod_{i=1}^{j-1}\left(1-\boldsymbol{v}_{i}\right)$ for $j=2,3, \ldots$, and let $\boldsymbol{\theta}_{1}, \boldsymbol{\theta}_{2}, \ldots \stackrel{\text { iid }}{\sim} P_{0}$ where the probability distribution $P_{0}$ is called the base distribution. Then the discrete random probability measure

$$
\boldsymbol{P}=\sum_{j=1}^{\infty} \boldsymbol{\pi}_{j} \delta_{\boldsymbol{\theta}_{j}}
$$

is distributed according to the Pitman-Yor process denoted by $\operatorname{PY}\left(\alpha, d, P_{0}\right)$. Due to its discrete nature (1), the Pitman-Yor process creates ties with positive probability among data $\boldsymbol{x}_{1}, \ldots, \boldsymbol{x}_{n}$ generated from the Bayesian model

$$
\begin{gathered}
\boldsymbol{x}_{1}, \ldots, \boldsymbol{x}_{n} \mid \boldsymbol{P} \stackrel{\mathrm{iid}}{\sim} \boldsymbol{P}, \\
\boldsymbol{P} \sim \operatorname{PY}\left(\alpha, d, P_{0}\right) .
\end{gathered}
$$

Assuming that the base distribution is non-atomic, the distribution on random partitions $\boldsymbol{C}$ of the first $n$ integers $\{1, \ldots, n\}$ induced by the data $\boldsymbol{x}_{1}, \ldots, \boldsymbol{x}_{n}$ is

$$
\mathbb{P}(\boldsymbol{C}=C)=\frac{d^{|C|}}{(\alpha)_{(n)}}\left(\frac{\alpha}{d}\right)_{(|C|)} \prod_{c \in C}(1-d)_{(|c|-1)},
$$

where $(x)_{(n)}=x(x+1) \ldots(x+n-1)$ denotes the rising factorial. The multiplicative factor before the product in (2) is also commonly (and equivalently) written as $\left(\prod_{i=1}^{|C|-1} \alpha+i d\right) /(\alpha+1)_{(n-1)}$ in the literature. When the discount parameter $d$ is set to zero, the Pitman-Yor process reduces to the Dirichlet process and the partition distribution (2) boils down to the celebrated Chinese Restaurant process [CRP, see 1]. By abuse of language, we call the partition distribution (2) the Pitman-Yor's CRP. Under the latter partition distribution, the number of parts in a partition $C$ of $n$ elements, $|C|$, grows to infinity as a power-law of the sample size, $n^{d}$ [see 29, for details]. This Pitman-Yor power-law growth is more in tune with most of empirical data [8] than the logarithmic growth $\alpha \log n$ induced by the Dirichlet process CRP.

The purpose of this note is to provide a simple proof of Pitman-Yor's CRP (2) from its stick-breaking representation (1) (Theorem 2.1). This generalizes the derivation by [22] who obtained the Dirichlet process CRP [1] from Sethuraman's stick-breaking representation [33]. Although the general proof technique for Theorem 2.1 proceeds along similar lines to that of [22], most of the intermediate results required for the proof rely on different techniques or are novel (such as Lemma 3.2). We also provide in Proposition 2.2 the marginal distribution of the latent variables that identify the observations clusters (so-called allocation variables).

A natural extension to the Dirichlet and Pitman-Yor processes are Gibbs-type priors [9]. They are characterized by a simple CRP representation

$$
\mathbb{P}(\boldsymbol{C}=C)=V_{n, k} \prod_{c \in C}(1-d)_{(|c|-1)},
$$

where the triangular array of nonnegative parameters $V_{n, k}, 1 \leq k \leq n$, satisfy the backward recurrence relation

$$
V_{n, k}=(n-d k) V_{n+1, k}+V_{n+1, k+1} \text {, }
$$

with $V_{1,1}=1$. Like the Dirichlet and Pitman-Yor processes, Gibbs-type priors have an explicit stick-breaking representation. See for instance Theorem 14.23 of [16] for details. But in contrast to the Dirichlet and PitmanYor cases, the latter representation is obtained by breaking sequentially proportions of a stick of length one in a non independent manner. Due to this loss of independence, we have not been able to extend the present proof to the class of Gibbs-type priors, which remains an open problem. 


\section{Partition distribution from stick-breaking}

We denote the set of positive integers $\{1,2, \ldots\}$ by $\mathbb{N}$. For $n \in \mathbb{N}$, we say that integers $z_{1}, \ldots, z_{n} \in \mathbb{N}$ induce a partition of $\{1, \ldots, n\}$ denoted by $C$ into disjoint and non-empty parts $c_{1}, \ldots, c_{k}$ if $C$ is induced by the equivalence relation $z_{i}=z_{j}$, for any $i, j \in\{1, \ldots, n\}$. We denote by $k$ the number of parts $|C|$ in the partition $C$ and by $\left|c_{j}\right|$ the number of elements in part $c_{j}$. Bold font is used to represent random variables. We denote by $\Gamma$ the gamma function and by $B$ the beta function.

Theorem 2.1. Suppose

$$
\begin{aligned}
& \boldsymbol{v}_{i} \stackrel{\text { ind }}{\sim} \operatorname{Beta}(1-d, \alpha+i d) \text { for } i=1,2, \ldots, \\
& \boldsymbol{\pi}_{j}=\boldsymbol{v}_{j} \prod_{i=1}^{j-1}\left(1-\boldsymbol{v}_{i}\right) \text { for } j=1,2, \ldots
\end{aligned}
$$

Let allocation variables be defined by

$$
\boldsymbol{z}_{1}, \ldots, \boldsymbol{z}_{n} \mid \boldsymbol{\pi}=\pi \stackrel{\mathrm{iid}}{\sim} \pi, \text { meaning, } \mathbb{P}\left(\boldsymbol{z}_{i}=j \mid \pi\right)=\pi_{j},
$$

and $\boldsymbol{C}$ denote the random partition of $\{1, \ldots, n\}$ induced by $z_{1}, \ldots, z_{n}$. Then

$$
\mathbb{P}(\boldsymbol{C}=C)=\frac{d^{|C|}}{(\alpha)_{(n)}}\left(\frac{\alpha}{d}\right)_{(|C|)} \prod_{c \in C}(1-d)_{(|c|-1)}
$$

The proof of Theorem 2.1 follows the lines of [22]'s derivation. We need the next two technical results, which we will prove in Section 3. Let $C_{z}$ denote the partition $\{1, \ldots, n\}$ induced by $z$ for any $z \in \mathbb{N}^{n}$. Let $k$ be the number of parts in the partition. We define $m(z)=\max \left\{z_{1}, \ldots, z_{n}\right\}$, and $g_{j}(z)=\#\left\{i: z_{i} \geq j\right\}$.

Proposition 2.2. For any $n \in \mathbb{N}$, any $z \in \mathbb{N}^{n}$, the marginal distribution of the allocation variables vector $\boldsymbol{z}=\left(\boldsymbol{z}_{1}, \ldots, \boldsymbol{z}_{n}\right)$ is given $b y$

$$
\mathbb{P}(\boldsymbol{z}=z)=\frac{1}{(\alpha)_{(n)}} \prod_{c \in C_{z}} \frac{\Gamma(|c|+1-d)}{\Gamma(1-d)} \prod_{j=1}^{m(z)} \frac{\alpha+(j-1) d}{g_{j}(z)+\alpha+(j-1) d} .
$$

Lemma 2.3. For any $n \in \mathbb{N}$, any partition $C$ of $\{1, \ldots, n\}$,

$$
\sum_{z \in \mathbb{N}^{n}} \mathbb{1}\left(C_{z}=C\right) \prod_{j=1}^{m(z)} \frac{\alpha+(j-1) d}{g_{j}(z)+\alpha+(j-1) d}=\frac{d^{|C|}}{\prod_{c \in C}(|c|-d)}\left(\frac{\alpha}{d}\right)_{(|C|)} .
$$

Proof of Theorem 2.1.

$$
\begin{aligned}
\mathbb{P}(\boldsymbol{C}=C) & =\sum_{z \in \mathbb{N}^{n}} \mathbb{P}(\boldsymbol{C}=C \mid \boldsymbol{z}=z) \mathbb{P}(\boldsymbol{z}=z) \\
& \stackrel{(\mathrm{a})}{=} \sum_{z \in \mathbb{N}^{n}} \mathbb{1}\left(C_{z}=C\right) \frac{1}{(\alpha)_{(n)}}\left(\prod_{c \in C_{z}} \frac{\Gamma(|c|+1-d)}{\Gamma(1-d)}\right) \prod_{j=1}^{m(z)} \frac{\alpha+(j-1) d}{g_{j}(z)+\alpha+(j-1) d} \\
& =\frac{1}{(\alpha)_{(n)}}\left(\prod_{c \in C} \frac{\Gamma(|c|+1-d)}{\Gamma(1-d)}\right) \sum_{z \in \mathbb{N}^{n}} \mathbb{1}\left(C_{z}=C\right) \prod_{j=1}^{m(z)} \frac{\alpha+(j-1) d}{g_{j}(z)+\alpha+(j-1) d} \\
& \stackrel{(\mathrm{b})}{=} \frac{1}{(\alpha)_{(n)}}\left(\prod_{c \in C} \frac{\Gamma(|c|+1-d)}{\Gamma(1-d)}\right) \frac{d^{|C|}}{\prod_{c \in C}(|c|-d)}\left(\frac{\alpha}{d}\right)_{(|C|)} \\
& \stackrel{(\mathrm{c})}{=} \frac{1}{(\alpha)_{(n)}}\left(\prod_{c \in C}(1-d)_{(|c|-1)}\right)\left(\prod_{c \in C}(|c|-d)\right) \frac{d^{|C|}}{\prod_{c \in C}(|c|-d)}\left(\frac{\alpha}{d}\right)_{(|C|)}
\end{aligned}
$$




$$
=\frac{d^{|C|}}{(\alpha)_{(n)}}\left(\frac{\alpha}{d}\right)_{(|C|)} \prod_{c \in C}(1-d)_{(|c|-1)}
$$

where (a) is by Proposition 2.2, (b) is by Lemma 2.3, and (c) is since $\Gamma(|c|+1-d)=(|c|-d) \Gamma(|c|-d)$.

\section{Proofs of the technical results}

\subsection{Additional lemmas}

We require the following additional lemmas. Let $S_{k}$ denote the set of $k$ ! permutations of [k]. The following lemma is key for proving Lemma 2.3.

Lemma 3.1. For any $n_{1}, \ldots, n_{k} \in \mathbb{N}$,

$$
\sum_{\sigma \in S_{k}} \prod_{i=1}^{k} \frac{1}{a_{i}(\sigma)-(k-i+1) d}=\frac{1}{\prod_{i=1}^{k}\left(n_{i}-d\right)}
$$

where $a_{i}(\sigma)=n_{\sigma_{i}}+n_{\sigma_{i+1}}+\cdots+n_{\sigma_{k}}$.

The proof of Lemma 3.1 follows similar lines to that of Lemma 3.1 by [22], relying on an urn argument for simplifying sums over permutations in $S_{k}$. The latter lemma is stated and proven by [22] for integer-valued ball sizes $n_{1}, \ldots, n_{k} \in \mathbb{N}$, however it is straightforward to check that it carries over to any positive real-valued ball sizes. Application of this result to ball sizes $n_{1}-d, \ldots, n_{k}-d$, which are all positive since $d<1$ and $n_{j} \geq 1$ for any $j=1, \ldots, k$, yields the statement of Lemma 3.1.

Lemma 3.2. Let $b_{0}=0$ and introduce the notation $\bar{b}_{i}=b_{0}+b_{1}+\cdots+b_{i}$ for positive integers $b_{j}$. Then

$$
\sum_{b_{1} \in \mathbb{N}} \frac{\left(\frac{\alpha}{d}\right)_{\left(b_{1}\right)}}{\left(\frac{a_{1}+\alpha}{d}\right)_{\left(b_{1}\right)}}\left(\sum_{b_{2} \in \mathbb{N}} \frac{\left(\frac{\alpha}{d}+b_{1}\right)_{\left(b_{2}\right)}}{\left(\frac{a_{2}+\alpha}{d}+b_{1}\right)_{\left(b_{2}\right)}}\left(\ldots\left(\sum_{b_{k} \in \mathbb{N}} \frac{\left(\frac{\alpha}{d}+\bar{b}_{k-1}\right)_{\left(b_{k}\right)}}{\left(\frac{a_{k}+\alpha}{d}+\bar{b}_{k-1}\right)_{\left(b_{k}\right)}}\right) \cdots\right)\right)=\frac{\left(\frac{\alpha}{d}\right)_{(k)}}{\prod_{i=1}^{k}\left(\frac{a_{i}}{d}-(k-i+1)\right)}
$$

Proof. For $j \in\{1, \ldots, k\}$, let $A_{j}$ denote the function from $\mathbb{N}^{j-1}$ to $\mathbb{R}_{+}$defined by the partial product of the $k-j+1$ inner terms in the left-hand side of the lemma's statement,

$$
A_{j}\left(b_{1}, \ldots, b_{j-1}\right)=\sum_{b_{j} \in \mathbb{N}} \frac{\left(\frac{\alpha}{d}+\bar{b}_{j-1}\right)_{\left(b_{j}\right)}}{\left(\frac{a_{2}+\alpha}{d}+\bar{b}_{j-1}\right)_{\left(b_{j}\right)}}\left(\ldots\left(\sum_{b_{k} \in \mathbb{N}} \frac{\left(\frac{\alpha}{d}+\bar{b}_{k-1}\right)_{\left(b_{k}\right)}}{\left(\frac{a_{k}+\alpha}{d}+\bar{b}_{k-1}\right)_{\left(b_{k}\right)}}\right) \ldots\right) .
$$

We show by induction decreasing from $j=k$ to $j=1$ that the relation

$$
A_{j}\left(b_{1}, \ldots, b_{j-1}\right)=\frac{\left(\frac{\alpha}{d}+\bar{b}_{j-1}\right)_{(k-j+1)}}{\prod_{i=j}^{k}\left(\frac{a_{i}}{d}-(k-i+1)\right)}
$$

holds for any $j \in\{1, \ldots, k\}$, and that the relations obtained with $j=1$ corresponds to the statement of the lemma. When $j=k$, defining $X \sim \operatorname{Beta}\left(\frac{\alpha}{d}+\bar{b}_{k-1}, \frac{a_{k}}{d}\right)$ yields

$$
\begin{aligned}
A_{k}\left(b_{1}, \ldots, b_{k-1}\right) & =\sum_{b_{k} \in \mathbb{N}} \frac{\left(\frac{\alpha}{d}+\bar{b}_{k-1}\right)_{\left(b_{k}\right)}}{\left(\frac{a_{k}+\alpha}{d}+\bar{b}_{k-1}\right)_{\left(b_{k}\right)}} \stackrel{(\mathrm{a})}{=} \sum_{b_{k} \in \mathbb{N}} \mathbb{E}\left[X^{b_{k}}\right] \\
& =\mathbb{E}\left[\sum_{b_{k} \in \mathbb{N}} X^{b_{k}}\right] \stackrel{(\mathrm{b})}{=} \mathbb{E}\left[\frac{X}{1-X}\right] \stackrel{(\mathrm{a})}{=} \frac{\alpha+d \bar{b}_{k-1}}{a_{k}-d}=\frac{\left(\frac{\alpha}{d}+\bar{b}_{k-1}\right)_{(1)}}{\frac{a_{k}}{d}-1},
\end{aligned}
$$


where both steps (a) are due to the fact that if $X \sim \operatorname{Beta}(a, b)$, for $a, b>0$, then for $c>-a, d>-b$,

$$
\mathbb{E}\left[X^{c}(1-X)^{d}\right]=\frac{B(a+c, b+d)}{B(a, b)},
$$

while step (b) is due to the identity

$$
(1-X)\left(1+X+X^{2}+\cdots\right)=1 \text { when } X \in[0,1) \text { almost surely. }
$$

This proves the initialization for (5) with $j=k$. We now consider the case of an arbitrary $j \in\{1, \ldots, k-1\}$, and assume by induction hypothesis that Equation (5) holds for $j+1$, that is

$$
A_{j+1}\left(b_{1}, \ldots, b_{j}\right)=\frac{\left(\frac{\alpha}{d}+\bar{b}_{j}\right)_{(k-j)}}{\prod_{i=j+1}^{k}\left(\frac{a_{i}}{d}-(k+1-i)\right)} .
$$

Therefore, by definition of function $A_{j}$ in (4) and by induction hypothesis,

$$
A_{j}\left(b_{1}, \ldots, b_{j-1}\right)=\sum_{b_{j} \in \mathbb{N}} \frac{\left(\frac{\alpha}{d}+\bar{b}_{j-1}\right)_{\left(b_{j}\right)}}{\left(\frac{a_{j}+\alpha}{d}+\bar{b}_{j-1}\right)_{\left(b_{j}\right)}} A_{j+1}\left(b_{1}, \ldots, b_{j}\right)=\sum_{b_{j} \in \mathbb{N}} \frac{\left(\frac{\alpha}{d}+\bar{b}_{j-1}\right)_{\left(b_{j}\right)}}{\left(\frac{a_{j}+\alpha}{d}+\bar{b}_{j-1}\right)_{\left(b_{j}\right)}} \frac{\left(\frac{\alpha}{d}+\bar{b}_{j}\right)_{(k-j)}}{\prod_{i=j+1}^{k}\left(\frac{a_{i}}{d}-(k+1-i)\right)} .
$$

Rearranging the rising factorials in the numerator, we can write

$$
\begin{aligned}
\left(\frac{\alpha}{d}+\bar{b}_{j-1}\right)_{\left(b_{j}\right)}\left(\frac{\alpha}{d}+\bar{b}_{j}\right)_{(k-j)} & =\left(\frac{\alpha}{d}+\bar{b}_{j-1}\right)_{\left(b_{j}\right)}\left(\frac{\alpha}{d}+\bar{b}_{j-1}+b_{j}\right)_{(k-j)} \\
& =\left(\frac{\alpha}{d}+\bar{b}_{j-1}\right)_{\left(b_{j}+k-j\right)} \\
& =\left(\frac{\alpha}{d}+\bar{b}_{j-1}\right)_{(k-j)}\left(\frac{\alpha}{d}+\bar{b}_{j-1}+k-j\right)_{\left(b_{j}\right)},
\end{aligned}
$$

and thus factorize the terms independent of $b_{j}$ in order to obtain

$$
A_{j}\left(b_{1}, \ldots, b_{j-1}\right)=\frac{\left(\frac{\alpha}{d}+\bar{b}_{j-1}\right)_{(k-j)}}{\prod_{i=j+1}^{k}\left(\frac{a_{i}}{d}-(k+1-i)\right)} \sum_{b_{j} \in \mathbb{N}} \frac{\left(\frac{\alpha}{d}+\bar{b}_{j-1}+k-j\right)_{\left(b_{j}\right)}}{\left(\frac{a_{j}+\alpha}{d}+\bar{b}_{j-1}\right)_{\left(b_{j}\right)}} .
$$

The sum above can be rewritten, using $X \sim \operatorname{Beta}\left(\frac{\alpha}{d}+\bar{b}_{j-1}+k-j, \frac{a_{j}}{d}-(k-j)\right)$ and expressions (6) and (7), as

$$
\sum_{b_{j} \in \mathbb{N}} \mathbb{E}\left[X^{b_{j}}\right]=\mathbb{E}\left[\frac{X}{1-X}\right]=\frac{\frac{\alpha}{d}+\bar{b}_{j-1}+k-j}{\frac{a_{j}}{d}-(k+1-j)}
$$

Putting this all together,

$$
A_{j}\left(b_{1}, \ldots, b_{j-1}\right)=\frac{\left(\frac{\alpha}{d}+\bar{b}_{j-1}\right)_{(k-j)}}{\prod_{i=j+1}^{k}\left(\frac{a_{i}}{d}-(k+1-i)\right)} \frac{\frac{\alpha}{d}+\bar{b}_{j-1}+k-j}{\frac{a_{j}}{d}-(k+1-j)}=\frac{\left(\frac{\alpha}{d}+\bar{b}_{j-1}\right)_{(k-j+1)}}{\prod_{i=j}^{k}\left(\frac{a_{i}}{d}-(k+1-i)\right)}
$$

which coincides with (5) and proves the desired result for $j$. By induction, this result is true for all $j \in$ $\{1, \ldots, k\}$. Letting $j=1$ gives the result stated in the lemma, since $\bar{b}_{0}=b_{0}=0$.

\subsection{Proof of Proposition 2.2 and Lemma 2.3}

Proof of Proposition 2.2. For simplicity, we fix the allocation variable vector to a value $z$ and denote $m(z)$ by $m$ and $g_{j}(z)$ by $g_{j}$. We have

$$
\mathbb{P}\left(\boldsymbol{z}=z \mid \pi_{1}, \ldots, \pi_{m}\right)=\prod_{i=1}^{n} \pi_{z_{i}}=\prod_{j=1}^{m} \pi_{j}^{e_{j}}
$$

where $e_{j}=\#\left\{i: z_{i}=j\right\}$. Thus,

$$
\mathbb{P}\left(\boldsymbol{z}=z \mid v_{1}, \ldots, v_{m}\right)=\prod_{j=1}^{m}\left(v_{j} \prod_{i=1}^{j-1}\left(1-v_{i}\right)\right)^{e_{j}}=\prod_{j=1}^{m} v_{j}^{e_{j}}\left(1-v_{j}\right)^{f_{j}}
$$


where $f_{j}=\#\left\{i: z_{i}>j\right\}$. Therefore,

$$
\begin{aligned}
\mathbb{P}(\boldsymbol{z}=z) & =\int \mathbb{P}\left(\boldsymbol{z}=z \mid v_{1}, \ldots, v_{m}\right) p\left(v_{1}, \ldots, v_{m}\right) \mathrm{d} v_{1} \cdots \mathrm{d} v_{m} \\
& =\int\left(\prod_{j=1}^{m} v_{j}^{e_{j}}\left(1-v_{j}\right)^{f_{j}}\right) p_{1}\left(v_{1}\right) \cdots p_{m}\left(v_{m}\right) \mathrm{d} v_{1} \cdots \mathrm{d} v_{m} \\
& =\prod_{j=1}^{m} \int v_{j}^{e_{j}}\left(1-v_{j}\right)_{j}^{f_{j}} p_{j}\left(v_{j}\right) \mathrm{d} v_{j} \\
& \stackrel{(\mathrm{a})}{=} \prod_{j=1}^{m} \frac{B\left(e_{j}+1-d, f_{j}+\alpha+j d\right)}{B(1-d, \alpha+j d)} \\
& =\prod_{j=1}^{m} \frac{\Gamma\left(e_{j}+1-d\right) \Gamma\left(f_{j}+\alpha+j d\right)}{\Gamma\left(e_{j}+f_{j}+\alpha+(j-1) d+1\right)} \frac{\Gamma(\alpha+(j-1) d+1)}{\Gamma(1-d) \Gamma(\alpha+j d)} \\
& \stackrel{(\mathrm{b})}{=}\left(\prod_{j=1}^{m} \frac{\Gamma\left(e_{j}+1-d\right)}{\Gamma(1-d)}\right)\left(\prod_{j=1}^{m} \frac{\Gamma\left(g_{j+1}+\alpha+j d\right)}{\Gamma\left(g_{j}+\alpha+(j-1) d+1\right)}\right)\left(\prod_{j=1}^{m} \frac{\Gamma(\alpha+(j-1) d+1)}{\Gamma(\alpha+j d)}\right) \\
& \stackrel{(\mathrm{c})}{=}\left(\prod_{j=1}^{m} \frac{\Gamma\left(e_{j}+1-d\right)}{\Gamma(1-d)}\right)\left(\prod_{j=1}^{m} \frac{\alpha+(j-1) d}{g_{j}+\alpha+(j-1) d}\right) \frac{\Gamma\left(g_{m+1}+\alpha+m d\right) \Gamma(\alpha)}{\Gamma\left(g_{1}+\alpha\right) \Gamma(\alpha+m d)} \\
& \stackrel{(\mathrm{d})}{=} \frac{\Gamma(\alpha)}{\Gamma(n+\alpha)}\left(\prod_{c \in C_{z}} \frac{\Gamma(|c|+1-d)}{\Gamma(1-d)}\right)\left(\prod_{j=1}^{m} \frac{\alpha+(j-1) d}{g_{j}+\alpha+(j-1) d}\right)
\end{aligned}
$$

where step (a) follows from Equation 6, step (b) since $f_{j}=g_{j+1}$ and $g_{j}=e_{j}+f_{j}$, step (c) since $\Gamma(x+1)=x \Gamma(x)$, and step (d) since $g_{1}=n$ and $g_{m+1}=0$.

Proof of Lemma 2.3. For given $n$ and $C$, let $k=|C|$, and denote the parts of $C$ by $c_{1}, \ldots, c_{k}$. We denote the distinct values taken on by $z_{1}, \ldots, z_{n}$ by $j_{1}<\cdots<j_{k}$. We define $j_{0}=b_{0}=0, b_{i}=j_{i}-j_{i-1}$, and $\bar{b}_{i}=b_{0}+\cdots+b_{i}$ for $i \in\{1, \ldots, k\}$. We use the notation $a_{i}(\sigma)=\left|c_{\sigma_{i}}\right|+\cdots+\left|c_{\sigma_{k}}\right|$, where $\sigma$ is the permutation of $[k]$ such that $c_{\sigma_{i}}=\left\{\ell: z_{\ell}=j_{i}\right\}$. Then for any $z \in \mathbb{N}^{n}$ such that $C_{z}=C$,

$$
\prod_{j=1}^{m(z)} \frac{\alpha+(j-1) d}{g_{j}(z)+\alpha+(j-1) d}=\prod_{j=1}^{m(z)} \frac{\frac{\alpha}{d}+j-1}{\frac{g_{j}(z)+\alpha}{d}+j-1}=\prod_{i=1}^{k} \prod_{j=\bar{b}_{i-1}+1}^{\bar{b}_{i}} \frac{\frac{\alpha}{d}+j-1}{\frac{g_{j}(z)+\alpha}{d}+j-1}=\prod_{i=1}^{k} \frac{\left(\frac{\alpha}{d}+\bar{b}_{i-1}\right)_{\left(b_{i}\right)}}{\left(\frac{\alpha+a_{i}(\sigma)}{d}+\bar{b}_{i-1}\right)_{\left(b_{i}\right)}},
$$

because $g_{j}(z)=a_{i}(\sigma)$ for $\bar{b}_{i-1}<j \leq \bar{b}_{i}$. It follows from the definition of $b=\left(b_{1}, \ldots, b_{k}\right)$ and $\sigma$ that there is a one-to-one correspondence between $\left\{z \in \mathbb{N}^{n}: C_{z}=C\right\}$ and $\left\{(\sigma, b): \sigma \in S_{k}, b \in \mathbb{N}^{k}\right\}$. Therefore,

$$
\begin{array}{rl}
\sum_{z \in \mathbb{N}^{n}} & \mathbb{1}\left(C_{z}=C\right) \prod_{j=1}^{m(z)} \frac{\alpha+(j-1) d}{g_{j}(z)+\alpha+(j-1) d} \\
\quad & \sum_{\sigma \in S_{k}}\left(\sum_{b_{1} \in \mathbb{N}} \frac{\left(\frac{\alpha}{d}+\bar{b}_{0}\right)_{\left(b_{1}\right)}}{\left(\frac{a_{1}+\alpha}{d}+\bar{b}_{0}\right)_{\left(b_{1}\right)}}\left(\ldots\left(\sum_{b_{k} \in \mathbb{N}} \frac{\left(\frac{\alpha}{d}+\bar{b}_{k-1}\right)_{\left(b_{k}\right)}}{\left(\frac{a_{k}+\alpha}{d}+\bar{b}_{k-1}\right)_{\left(b_{k}\right)}}\right) \ldots\right)\right) \\
& \stackrel{(\mathrm{a})}{=} \sum_{\sigma \in S_{k}} \prod_{i=1}^{k} \frac{\left(\frac{\alpha}{d}\right)_{(k)}}{\frac{a_{i}(\sigma)}{d}-(k-i+1)} \\
= & d^{k}\left(\frac{\alpha}{d}\right)_{(k)} \sum_{\sigma \in S_{k}} \prod_{i=1}^{k} \frac{1}{a_{i}(\sigma)-(k-i+1) d} \\
& \stackrel{(\mathrm{b})}{=} \frac{d^{k}}{\prod_{c \in C}(|c|-d)}\left(\frac{\alpha}{d}\right)_{(k)},
\end{array}
$$

where step (a) follows from Lemma 3.2 and step (b) from Lemma 3.1. 
Acknowledgement: The authors would like to thank two anonymous referees for helpful suggestions that improved the presentation of the paper and especially the proof of Lemma 3.2, as well as Bernardo Nipoti for fruitful discussions that initiated this work.

\section{References}

[1] Antoniak, C. E. (1974). Mixtures of Dirichlet processes with applications to Bayesian nonparametric problems. Ann. Statist. 2(6), 1152-1174.

[2] Arbel, J., P. De Blasi, and I. Prünster (2018). Stochastic approximations to the Pitman-Yor process. Bayesian Anal., to appear. Available at https://doi.org/10.1214/18-BA1127.

[3] Arbel, J., S. Favaro, B. Nipoti, and Y. W. Teh (2017). Bayesian nonparametric inference for discovery probabilities: credible intervals and large sample asymptotics. Statist. Sinica 27(2), 839-858.

[4] Bassetti, F., R. Casarin, and F. Leisen (2014). Beta-product dependent Pitman-Yor processes for Bayesian inference. J. Econometrics 180(1), 49-72.

[5] Battiston, M., S. Favaro, D. M. Roy, and Y. W. Teh (2018). A characterization of product-form exchangeable feature probability functions. Ann. Appl. Probab. 28(3), 1423-1448.

[6] Canale, A., A. Lijoi, B. Nipoti, and I. Prünster (2017). On the Pitman-Yor process with spike and slab base measure. Biometrika 104(3), 681-697.

[7] Caron, F., W. Neiswanger, F. Wood, A. Doucet, and M. Davy (2017). Generalized Pólya urn for time-varying Pitman-Yor processes. J. Mach. Learn. Res. 18(27), 1-32.

[8] Clauset, A., C. R. Shalizi, and M. E. Newman (2009). Power-law distributions in empirical data. SIAM Rev. 51(4), 661-703.

[9] De Blasi, P., S. Favaro, A. Lijoi, R. H. Mena, I. Prünster, and M. Ruggiero (2015). Are Gibbs-type priors the most natural generalization of the Dirichlet process? IEEE Trans. Pattern Anal. Mach. Intell. 37(2), 212-229.

[10] De Luca, G. and P. Zuccolotto (2011). A tail dependence-based dissimilarity measure for financial time series clustering. Adv. Data Anal. Classif. 5(4), 323-340.

[11] Derrida, B. (1981). Random-energy model: An exactly solvable model of disordered systems. Phys. Rev. B 24(5), $2613-2626$.

[12] Favaro, S., A. Lijoi, R. Mena, and I. Prünster (2009). Bayesian non-parametric inference for species variety with a twoparameter Poisson-Dirichlet process prior. J. R. Stat. Soc. Ser. B. Stat. Methodol. 71(5), 993-1008.

[13] Favaro, S. and S. G. Walker (2013). Slice sampling $\sigma$-stable Poisson-Kingman mixture models. J. Comput. Graph. Statist. 22(4), 830-847.

[14] Feng, S. and W. Sun (2010). Some diffusion processes associated with two parameter Poisson-Dirichlet distribution and Dirichlet process. Probab. Theory Relat. Fields 148(3-4), 501-525.

[15] Ferguson, T. S. (1973). A Bayesian analysis of some nonparametric problems. Ann. Statist. 1(2), 209-230.

[16] Ghosal, S. and A. Van der Vaart (2017). Fundamentals of Nonparametric Bayesian Inference. Cambridge University Press.

[17] Ishwaran, H. and L. F. James (2001). Gibbs sampling methods for stick-breaking priors. J. Amer. Statist. Assoc. 96(453), 161-173.

[18] Jara, A., E. Lesaffre, M. De lorio, and F. Quintana (2010). Bayesian semiparametric inference for multivariate doubly-intervalcensored data. Ann. Appl. Stat. 4(4), 2126-2149.

[19] Kerov, S. V. (2006). Coherent random allocations, and the Ewens-Pitman formula. J. Math. Sci. 138(3), 5699-5710.

[20] Kosmidis, I. and D. Karlis (2016). Model-based clustering using copulas with applications. Stat. Comput. 26(5), $1079-1099$.

[21] Lo, A. Y. (1984). On a class of Bayesian nonparametric estimates: I. Density estimates. Ann. Statist. 12(1), 351-357.

[22] Miller, J. W. (2019). An elementary derivation of the Chinese restaurant process from Sethuraman's stick-breaking process. Statist. Probab. Lett. 146, 112-117.

[23] Miller, J. W. and M. T. Harrison (2014). Inconsistency of Pitman-Yor process mixtures for the number of components. J. Mach. Learn. Res. 15(1), 3333-3370.

[24] Navarrete, C., F. A. Quintana, and P. Müller (2008). Some issues in nonparametric Bayesian modeling using species sampling models. Stat. Model. 8(1), 3-21.

[25] Ni, Y., P. Müller, Y. Zhu, and Y. Ji (2018). Heterogeneous reciprocal graphical models. Biometrics 74(2), 606-615.

[26] Perman, M., J. Pitman, and M. Yor (1992). Size-biased sampling of Poisson point processes and excursions. Probab. Theory Relat. Fields 92(1), 21-39.

[27] Petrov, L. A. (2009). Two-parameter family of infinite-dimensional diffusions on the Kingman simplex. Funct. Anal. Appl. 43(4), 279-296.

[28] Pitman, J. (1995). Exchangeable and partially exchangeable random partitions. Probab. Theory Relat. Fields 102(2), 145-158.

[29] Pitman, J. (2003). Poisson-Kingman partitions. In Statistics and Science: a Festschrift for Terry Speed, pp.1-34. IMS, Beachwood $\mathrm{OH}$.

[30] Pitman, J. and M. Yor (1997). The two-parameter Poisson-Dirichlet distribution derived from a stable subordinator. Ann. Probab. 25(2), 855-900. 
[31] Scarpa, B. and D. B. Dunson (2009). Bayesian hierarchical functional data analysis via contaminated informative priors. Biometrics 65(3), 772-780.

[32] Scricciolo, C. (2014). Adaptive Bayesian density estimation in $L^{p}$-metrics with Pitman-Yor or normalized inverse-Gaussian process kernel mixtures. Bayesian Anal. 9(2), 475-520.

[33] Sethuraman, J. (1994). A constructive definition of Dirichlet priors. Statist. Sinica 4(2), 639-650.

[34] Sudderth, E. B. and M. I. Jordan (2009). Shared segmentation of natural scenes using dependent Pitman-Yor processes. In D. Koller, D. Schuurmans, Y. Bengio and L. Bottou (Eds.), Advances in Neural Information Processing Systems 21, pp. 1585-1592. Curran Associates, Red Hook NY.

[35] Teh, Y. W. (2006). A hierarchical Bayesian language model based on Pitman-Yor processes. In Proceedings of the 21st International Conference on Computational Linguistics and the 44th annual meeting of the Association for Computational Linguistics, pp. 985-992. Association for Computational Linguistics, Stroudsburg PA.

[36] Vershik, A., M. Yor, and N. Tsilevich (2004). On the Markov-Krein identity and quasi-invariance of the gamma process. J. Math. Sci. 121(3), 2303-2310.

[37] Wood, F., J. Gasthaus, C. Archambeau, L. James, and Y. W. Teh (2011). The sequence memoizer. Comm. ACM 54(2), 91-98. 